\title{
Geometry of polar wedges in Riesz spaces and super-replication prices in incomplete financial markets
}

\author{
Frank Oertel and Mark P. Owen
}

\begin{abstract}
This paper is devoted to a further generalisation of the main results in [5] including the representation of the weak super-replication price (cf. equation (1.6)). In addition to the already established weakening of the technical assumptions in [5] (cf. [24] and [25]), the main results in [5] can be still generalised by considering the geometric structure of the underlying problem (based on the properties of Riesz spaces and polar wedges therein). In Section 5 we show under which geometric conditions of the relevant sets the results still hold (cf. Theorem 5.3 and Corollary 5.5). In particular, we can completely remove the restrictive admissibility assumption and carry forward equation (1.4) to a larger class of wedges $K \subseteq L^{0}$ (cf. Corollary 5.5).
\end{abstract}

Mathematics Subject Classification (2000). 1B16, 46N10, $60 \mathrm{G} 44$.

Keywords. Super-replication, Incomplete markets, Contingent claims, Duality theory, Weak topologies, Riesz spaces.

\section{Introduction}

Although this paper primarily is written for a mathematical audience who need not have a detailed knowledge of mathematical finance (including the related terminology of stochastic analysis), we occasionally have to use some specific terminology which cannot be explained in detail here, due to the limitation of space. Therefore, we would like to refer the reader to the introductory overview references $[4,13,29,31]$ and the further references therein.

Firstly, let us revisit the ideal, non-realistic case, namely the case of a complete (financial) market. In a complete market, there is a unique arbitrage-free price of a given derivative security or contingent claim. Its payoff (i. e., its terminal value) is modelled as a random variable $X \in L^{1}(\mathbb{P})$, where $\mathbb{P}$ denotes the original 
(statistical) probability measure. Given a finite time horizon $T>0$ and a constant risk-free rate of interest $r>0$, the unique arbitrage-free price is then given by the expected discounted terminal value $\mathbb{E}_{\mathbb{Q}}[\exp (-r T) X]=\exp (-r T) \mathbb{E}_{\mathbb{Q}}[X]$ which is computed with respect to the risk-neutral probability measure $\mathbb{Q}$ under which the underlying asset's expected return equals $r$ (i. e., under which the discounted asset price behaves like a martingale) and which is equivalent to $\mathbb{P}$. Due to the $\mathbb{Q}$-martingale property (respectively the $\mathbb{Q}$-sigma martingale property) of the discounted marketed security price, $\mathbb{Q}$ is called an equivalent martingale measure $($ EMM) (respectively an equivalent sigma martingale measure (ESMM)).

However, due to the incorporation of features like price jumps, transaction costs and illiquidity, financial markets in general do not allow a complete replication of payoffs by trading in marketed securities. They are incomplete. The classic no-arbitrage theory of valuation in complete markets, based on the unique price of a self-financing replicating portfolio, is not adequate for non-replicable payoffs in incomplete markets, where a perfect risk transfer is not possible. In incomplete markets, there can be many ESMMs, and one may ask for additional boundary criteria to select a specific candidate $\mathbb{Q}^{*}$ of all available ESMMs in this market. The expectation $\mathbb{E}_{\mathbb{Q}^{*}}[\exp (-r T) X]$ is then the chosen extension of the risk-neutral price function.

More generally, in an incomplete market with finite time horizon $T$, the discounted price process of $d$ risky assets altogether is modelled as a (non-continuous) $\mathbb{R}^{d}$-valued semimartingale $S=\left(S_{t}\right)_{t \in[0, T]}$. Not every contingent claim $X$ can be replicated by a self-financing trading strategy. For such contingent claims there exists a whole interval of arbitrage-free prices, as opposed to the case of a complete market, where there exists a unique replication price, as roughly sketched above. An upper bound for this price interval is the super-replication price

$$
\bar{\pi}(X):=\inf \left\{x \in \mathbb{R}: \text { there is an admissible } H \text { such that } X \leq x+G_{T}(H)\right\},
$$

where $G_{T}(H):=\int_{0}^{T} H_{u} \mathrm{~d} S_{u}$ denotes the discounted cumulative gain or loss at the time horizon $T$. Recall that an $\mathbb{R}^{d}$-valued predictable process $H$ is called an admissible trading strategy (cf. $[9,12]$ ) if $H$ is $S$-integrable, and there exists a constant $c \geq 0$ such that for all $t \in[0, T]$,

$$
\int_{0}^{t} H_{u} \mathrm{~d} S_{u} \geq-c, \quad \mathbb{P}-\text { a.s. }
$$

Let

$$
M_{1}^{\operatorname{adm}}(\mathbb{P}):=\left\{\mathbb{Q} \ll \mathbb{P}: K^{\mathrm{adm}} \subseteq L^{1}(\mathbb{Q}) \text { and } \mathbb{E}_{\mathbb{Q}}[X] \leq 0 \text { for all } X \in K^{\mathrm{adm}}\right\},
$$

be the set of all separating measures, where $K^{\mathrm{adm}}:=\left\{G_{T}(H): H\right.$ is admissible $\}$ denotes the wedge of all terminal wealths originating from zero-financed admissible trading strategies (cf. Section 2 for the definition of a wedge).

Although we will not not make use of it in this paper, we would like to recall now some well-known representations of $M_{1}^{\mathrm{adm}}(\mathbb{P})$ which depend upon the 
regularity (boundedness) of the semimartingale $S$. The final statement was shown in the well-known article [11]:

Remark 1.1. (i) If $S$ is bounded then $M_{1}^{\operatorname{adm}}(\mathbb{P})$ is the set of all $\mathbb{P}$-absolutely continuous probability measures $\mathbb{Q}$ such that $S$ is a $\mathbb{Q}$-martingale.

(ii) If $S$ is locally bounded then $M_{1}^{\text {adm }}(\mathbb{P})=M^{a}(\mathbb{P})$, where $M^{a}(\mathbb{P})$ denotes the set of all $\mathbb{P}$-absolutely continuous probability measures $\mathbb{Q}$ such that $S$ is a $\mathbb{Q}$-local martingale.

(iii) In the general case (where $S$ may not be locally bounded), if $M_{1}^{\operatorname{adm}}(\mathbb{P}) \neq \emptyset$ then $M_{1}^{\operatorname{adm}}(\mathbb{P})$ is the closure, in the topology induced by the total variation norm, of the set $M_{\sigma}^{a}(\mathbb{P})$ of all $\mathbb{P}$-absolutely continuous probability measures $\mathbb{Q}$ such that $S$ is a $\mathbb{Q}$-sigma-martingale.

If $S$ is locally bounded and the set $M^{e}(\mathbb{P})$ of all $\mathbb{P}$-equivalent probability measures $\mathbb{Q}$ such that $S$ is a $\mathbb{Q}$-local martingale is non-empty, it is well-known (cf. [10]) that for $X$ bounded from below

$$
\bar{\pi}(X)=\sup _{\mathbb{Q} \in M^{e}(\mathbb{P})} \mathbb{E}_{\mathbb{Q}}[X] .
$$

In the case of a general semimartingale $S$, equation (1.3) holds if one substitutes the set $M^{e}(\mathbb{P})$ with the set $M_{\sigma}^{e}(\mathbb{P})$, provided $M_{\sigma}^{e}(\mathbb{P}) \neq \emptyset^{1}$ (cf. [11, Theorem 5.12]).

It is already useful at this point to extend the definition of the super-replication price to allow terminal wealths from an arbitrary fixed wedge $K$. Several candidates for $K$ may be appropriate including admissible strategies, acceptable strategies (cf. [18]) or permissible strategies (cf. [26]).

Let $X \in L^{0}$ and $K \subseteq L^{0}$ be an arbitrarily given wedge. Assume that $A(X ; K) \neq \emptyset$, where

$$
\begin{aligned}
A(X ; K): & =\{x \in \mathbb{R}: X \leq x+G \text { for some } G \in K\} \\
& =\left\{x \in \mathbb{R}: X-x \in K-L_{+}^{0}\right\},
\end{aligned}
$$

and consider

$$
\bar{\pi}(X ; K):=\inf (A(X ; K)) .
$$

Note that $\bar{\pi}\left(X ; K^{\mathrm{adm}}\right)=\bar{\pi}(X)$ if $A\left(X ; K^{\mathrm{adm}}\right) \neq \emptyset$ and that $\bar{\pi}\left(X ; K_{2}\right) \leq \bar{\pi}\left(X ; K_{1}\right)$ if $K_{1} \subseteq K_{2}$ and $A\left(X ; K_{1}\right) \neq \emptyset$.

Let us now assume that $M_{1}^{\mathrm{adm}}(\mathbb{P}) \neq \emptyset$. If one is interested in pricing the claim $X$ by using separating measures from the set $M_{1}^{\operatorname{adm}}(\mathbb{P})$, it is natural to assume that $X \in L\left(M_{1}^{\operatorname{adm}}(\mathbb{P})\right):=\bigcap_{\mathbb{Q} \in M_{1}^{\text {adm }}(\mathbb{P})} L^{1}(\mathbb{Q})$. For such $X$ we always have

$$
-\infty<\sup _{\mathbb{Q} \in M_{1}^{\operatorname{adm}}(\mathbb{P})} \mathbb{E}_{\mathbb{Q}}[X] \leq \bar{\pi}(X)<+\infty .
$$

Note also that by construction

$$
K^{\mathrm{adm}} \subseteq L\left(M_{1}^{\mathrm{adm}}(\mathbb{P})\right)
$$

\footnotetext{
${ }^{1}$ The condition $M_{\sigma}^{e}(\mathbb{P}) \neq \emptyset$ is equivalent to the (NFLVR) property (cf. [12, Theorem 3.4]).
} 
Moreover, an easy calculation shows that the set $L\left(M_{1}^{\operatorname{adm}}(\mathbb{P})\right)$ contains all contingent claims $X$ in $L^{0}$ which are bounded from below and satisfy $A\left(X ; K^{\mathrm{adm}}\right) \neq \emptyset$.

However, one can construct $Y \in L\left(M_{1}^{\mathrm{adm}}(\mathbb{P})\right)$ such that

$$
\sup _{\mathbb{Q} \in M_{1}^{\operatorname{adm}}(\mathbb{P})} \mathbb{E}_{\mathbb{Q}}[Y]<\bar{\pi}(Y),
$$

suggesting that the use of admissible trading strategies is unsuitable for super-replication of unbounded claims (cf. [5]); the wedge $K^{\text {adm }}$ is "too small" for the purpose of super-replicating such contingent claims.

Let $X \in L\left(M_{1}^{\operatorname{adm}}(\mathbb{P})\right)$. Then

$$
\bar{\pi}\left(X ; K^{\mathrm{adm}}\right)=\bar{\pi}\left(X ; s_{L\left(M_{1}^{\mathrm{adm}}(\mathbb{P})\right)}\left(K^{\mathrm{adm}}\right)\right),
$$

where

$$
\begin{aligned}
s_{L\left(M_{1}^{\operatorname{adm}}(\mathbb{P})\right)}\left(K^{\mathrm{adm}}\right) & =\left\{G \in L\left(M_{1}^{\mathrm{adm}}(\mathbb{P})\right): G \leq U \text { for some } U \in K^{\mathrm{adm}}\right\} \\
& =\bigcap_{\mathbb{Q} \in M_{1}^{\mathrm{adm}}(\mathbb{P})}\left(K^{\mathrm{adm}}-L_{+}^{1}(\mathbb{Q})\right)
\end{aligned}
$$

denotes the smallest wedge $C \subseteq L\left(M_{1}^{\text {adm }}(\mathbb{P})\right)$ such that $-L\left(M_{1}^{\text {adm }}(\mathbb{P})\right)+\subseteq C$ and $K^{\text {adm }} \subseteq C$ (cf. Definition 2.2 and (5.3)). The geometry of such umbrella cones will play an important role in this paper.

The following natural question was posed in [5]. Is it possible to find an enlarged wedge $C \in L\left(M_{1}^{\text {adm }}(\mathbb{P})\right)$, satisfying $s_{L\left(M_{1}^{\text {adm }}(\mathbb{P})\right)}\left(K^{\text {adm }}\right) \subseteq C$, and a suitable non-empty subset $M$ of $M_{1}^{\text {adm }}(\mathbb{P})$ (which may depend on $K^{\text {adm }}$ ), such that

$$
\bar{\pi}(X ; C)=\sup _{\mathbb{Q} \in M} \mathbb{E}_{\mathbb{Q}}[X] ?
$$

In the admissible case, a partial answer to the question was provided in [5], where preferences of the investor were incorporated in the construction of the enlarged wedge by means of the convex conjugate $\Phi$ of their utility function $U$. This wedge was defined as

$$
C_{\Phi}^{\mathrm{adm}}:=\bigcap_{\mathbb{Q} \in M_{\Phi}^{\operatorname{adm}}}{\overline{K^{\mathrm{adm}}-L_{+}^{1}(\mathbb{Q})}}^{L^{1}(\mathbb{Q})},
$$

where

$$
M_{\Phi}^{\mathrm{adm}}:=M_{\Phi}^{\mathrm{adm}}(\mathbb{P}):=\left\{\mathbb{Q} \in M_{1}^{\mathrm{adm}}(\mathbb{P}): \Phi\left(\frac{\mathrm{d} \mathbb{Q}}{\mathrm{d} \mathbb{P}}\right) \in L^{1}(\mathbb{P})\right\}
$$

denotes the set of pricing measures with finite entropy.

Under the assumptions that the utility function $U$ has the condition of Reasonable Asymptotic Elasticity at $-\infty$ and at $+\infty$ and is bounded from above, ${ }^{2}$ and if $\left\{\mathbb{Q} \in M_{\Phi}^{\text {adm }}: \mathbb{Q} \sim \mathbb{P}\right\} \neq \emptyset$, Biagini and Frittelli showed that

$$
\bar{\pi}\left(X ; C_{\Phi}^{\mathrm{adm}}\right)=\sup _{\mathbb{Q} \in M_{\Phi}^{\text {adm }}} \mathbb{E}_{\mathbb{Q}}[X] .
$$

${ }^{2}$ i.e., if $\Phi(0)=\lim _{x \rightarrow \infty} U(x)<\infty$ 
The scope of this paper is a further generalisation of the main results in [5] including equation (1.6).

In [24] some of the technical assumptions in [5] were weakened, in particular the condition that the utility function must be bounded from above. In [25] it was subsequently shown in detail that the condition of Reasonable Asymptotic Elasticity can be relaxed, provided the set of separating measures with finite entropy is replaced by the set of separating measures with finite loss-entropy. In the current paper we show that the main results in [5] can be still generalised by considering the pure geometric structure of the underlying problem (based on the properties of Riesz spaces) without explicitly referring to an underlying utility function or its conjugate. In particular, we investigate under which geometric conditions of the relevant sets $K, M, C$ the results still hold (for example, $M$ has to be a face of $M_{1}(\mathbb{P})$ ). Our main results, Theorem 5.3, respectively its geometric version, Theorem 5.4, together with Corollary 5.5 transmit Theorem 4, [6] (and Theorem 5, [5]) from wedges of attainable terminal wealths resulting from admissible trading strategies to arbitrary wedges $K$ in $L^{0}$ and arbitrary non-empty arbitrary faces $M$ of $M_{1} \equiv M_{1}(\mathbb{P} ; K)$.

Section 6 reflects how the main results in [5] can be derived as a special case of our geometric approach.

\section{Preliminaries and notations}

In this section, we introduce some basic notation and terminology which we will use throughout the paper. The scalar field for vector spaces is assumed to be the real field $\mathbb{R}$ only, and most of our notations and definitions from probability theory, convex analysis and functional analysis are standard. We refer the reader to the monographs $[17,19,33]$ for the necessary background in functional analysis, and recommend the monographs $[8,28]$ for the basics of convex analysis.

From [2], let us recall that a wedge in a real vector space $E$ is a non-empty convex set $C \subseteq E$ satisfying $\lambda C \subseteq C$ for all $\lambda \geq 0 .{ }^{3}$ Obviously, a non-empty subset $C$ of $E$ is a wedge if and only if it is closed under addition and non-negative scalar multiplication. Let $S$ be an arbitrary non-empty subset of the vector space $E$. Let $\mathcal{W}(S)$ denote the smallest wedge in $E$ which contains $S$, i.e., the wedge generated by $S$ (cf. [2]). It is easy to show that

$$
\mathcal{W}(S)=\{\lambda x: \lambda \geq 0, x \in \operatorname{co}(S)\}=\mathcal{W}(\operatorname{co}(S)),
$$

where $\operatorname{co}(S)$ denotes the convex hull of $S$. We therefore arrive at the following description of $[S]$, the linear span of $S$, which we shall use in the proof of Proposition 4.8:

$$
[S]=\mathcal{W}(S)-\mathcal{W}(S)
$$

${ }^{3}$ In the literature, a wedge quite often is denoted as a convex cone. 
Lemma 2.1. Let $E$ be a real vector space, $S \subseteq E$ be convex and $T \subseteq E$ be a wedge. If $S \cap T$ is non-empty then

$$
\mathcal{W}(S \cap T)=\mathcal{W}(S) \cap T .
$$

Proof. Since $\mathcal{W}(S) \cap T$ is a wedge which contains the non-empty set $S \cap T$, it follows immediately that

$$
\mathcal{W}(S \cap T) \subseteq \mathcal{W}(S) \cap T
$$

Therefore, without loss of generality, we may assume that $\mathcal{W}(S) \cap T \neq\{0\}$. So, let $x \in(\mathcal{W}(S) \cap T) \backslash\{0\}$. Since $0 \neq x \in \mathcal{W}(S)$, (2.1) implies that $x=\lambda w$, where $\lambda>0$ and $w \in \operatorname{co}(S)=S$. Since $x \in T$ and $T$ is a wedge, it consequently follows that $w=\lambda^{-1} x \in S \cap T \subseteq \mathcal{W}(S \cap T)$. Hence, $(\mathcal{W}(S) \cap T) \backslash\{0\} \subseteq \mathcal{W}(S \cap T)$, and the remaining inclusion follows.

In order to embed utility-based super-replication prices in a mathematically concise framework, it is very useful to work in vector lattices (or Riesz spaces). Standard references are for instance $[1,22,23,32]$.

Definition 2.2. Let $(E, \leq)$ be a vector lattice and $C \subseteq E$ a wedge in $E$. $C$ is an umbrella (wedge) in $E$ if $-E_{+}=\{x \in E: x \leq 0\} \subseteq C$.

Given an arbitrary wedge $K \subseteq E$, we denote by $s_{E}(K)$ the umbrella hull, i. e., the smallest umbrella in $E$ which contains $K$. Note that

$$
s_{E}(K)=\{x \in E: \exists g \in K \text { s.t. } x \leq g\}=K-E_{+} .
$$

Consequently, a wedge $C \subseteq E$ is an umbrella in $E$ if and only if $C=s_{E}(C)=$ $C-E_{+}$.

Proposition 2.3. Let $\left(E_{\alpha}\right)_{\alpha \in A}$ be a family of vector sublattices of a vector lattice L. Then $E:=\bigcap_{\alpha \in A} E_{\alpha}$ is a vector sublattice of $L$. Let $K \subseteq E$ be an arbitrary wedge in E. Then

$$
s_{E}(K)=\bigcap_{\alpha \in A} s_{E_{\alpha}}(K)=\bigcap_{\alpha \in A}\left(K-\left(E_{\alpha}\right)_{+}\right) .
$$

Proof. Let $C$ be an arbitrary umbrella wedge in $E$ such that $K \subseteq C$. Then

$$
\bigcap_{\alpha \in \mathrm{A}} s_{E_{\alpha}}(K) \subseteq \bigcap_{\alpha \in \mathrm{A}}\left(s_{E_{\alpha}}(K) \cap E\right) \subseteq \bigcap_{\alpha \in \mathrm{A}}\left(s_{E_{\alpha}}(C) \cap E\right) \subseteq \bigcap_{\alpha \in \mathrm{A}} s_{E}(C)=C .
$$

Hence, $\bigcap_{\alpha \in \mathrm{A}} s_{E_{\alpha}}(K) \subseteq s_{E}(K)$. The other inclusion is trivial.

Example 2.4. Assume that $M_{1}^{\operatorname{adm}}(\mathbb{P}) \neq \emptyset$. Set $E:=\bigcap_{\mathbb{Q} \in M_{1}^{\operatorname{adm}}(\mathbb{P})} L^{1}(\mathbb{Q})$. Then $K^{\mathrm{adm}} \subseteq E$, and

$$
s_{E}\left(K^{\mathrm{adm}}\right)=\bigcap_{\mathbb{Q} \in M_{1}^{\mathrm{adm}}(\mathbb{P})}\left(K^{\mathrm{adm}}-L_{+}^{1}(\mathbb{Q})\right) .
$$


Next, we apply basic duality theory, where we adopt the approach of [17]. To this end, let $(E, F)$ be an arbitrary bilinear system of real vector spaces. For a non-empty set $A \subseteq E$ we define its polar wedge $A^{\triangleleft} \subseteq F$ by

$$
A^{\triangleleft}:=\{w \in F:\langle z, w\rangle \leq 0 \quad \forall z \in A\} .
$$

For a non-empty set $B \subseteq F$, its polar wedge $B^{\triangleleft} \subseteq E$ can be defined similarly. Clearly, $A^{\triangleleft}$ is a wedge and $A^{\triangleleft} \subseteq A^{\circ}$, where $A^{\circ}:=\{w \in F:\langle z, w\rangle \leq 1 \quad \forall z \in A\}$ denotes the polar of $A$ (cf. [33, Section 0.7]).

Remark 2.5. Note that the definition of a polar is not handled univocally! Some authors prefer to define the polar of $A$ as the set $\{w \in F:|\langle z, w\rangle| \leq 1 \quad \forall z \in A\}$ (cf. e. g. [17]). However, if the set $A$ is circled, i. e., if $\{\lambda x: \lambda \in[-1,1], x \in A\} \subseteq A$, both definitions coincide. Throughout the article, we will use the definition above including the related version of the bipolar theorem (see [33, Theorem 0.8]).

If in addition $A$ is a wedge, it easily follows that $A^{\triangleleft}=A^{\circ}$. The next result shows why we call $A^{\triangleleft}$ a "polar wedge":

Proposition 2.6. Let $(E, F)$ be an arbitrary bilinear system of real vector spaces and $A$ be an arbitrary non-empty subset of $E$. Then:

$$
A^{\triangleleft}=(\mathcal{W}(A))^{\circ} \text {. }
$$

In particular, $A^{\triangleleft}$ is $\sigma(F, E)$-closed.

Consequently, a direct application of the bipolar theorem (see [33, Theorem 0.8]) leads to the following result which we shall use in the proof of Theorem 6.4:

Proposition 2.7. Let $(E, F)$ be an arbitrary bilinear system of real vector spaces and $A$ be an arbitrary non-empty subset of $E$. Then:

$$
A^{\triangleleft \triangleleft}=\overline{\mathcal{W}(A)}^{\sigma(E, F)} .
$$

In other words, $A \triangleleft \triangleleft$ is the smallest $\sigma(E, F)$-closed wedge which contains $A$. If in addition $A$ is a wedge, then $A^{\triangleleft \triangleleft}=\bar{A}^{\sigma(E, F)}$.

Lemma 2.8. Let $(E, F)$ be an arbitrary bilinear system and $\left(A_{\gamma}\right)_{\gamma \in \Gamma}$ be a family of non-empty subsets of $E$. Then

(i) $\left(\bigcup_{\gamma \in \Gamma} A_{\gamma}\right)^{\triangleleft}=\bigcap_{\gamma \in \Gamma} A_{\gamma}^{\triangleleft}$ and

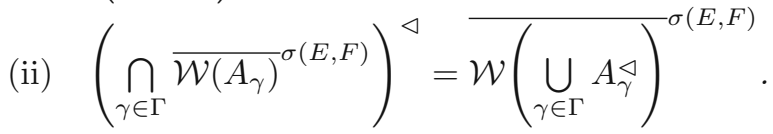

Proof. Statement (i) follows immediately from the definition of a polar wedge.

If we apply statement (i) to the family $\left(B_{\gamma}\right)_{\gamma \in \Gamma}$, where $B_{\gamma}:=A_{\gamma}^{\triangleleft}$, Proposition 2.7 implies that

$$
\left(\bigcap_{\gamma \in \Gamma} \overline{\mathcal{W}\left(A_{\gamma}\right)} \sigma(E, F)\right)^{\triangleleft}=\left(\bigcap_{\gamma \in \Gamma} B_{\gamma}^{\triangleleft}\right)^{\triangleleft} \stackrel{(i)}{=}\left(\bigcup_{\gamma \in \Gamma} B_{\gamma}\right)^{\triangleleft \triangleleft} .
$$


Again, due to Proposition 2.7, we obtain

$$
\left(\bigcup_{\gamma \in \Gamma} B_{\gamma}\right)^{\triangleleft \triangleleft} \overline{\mathcal{W}\left(\bigcup_{\gamma \in \Gamma} B_{\gamma}\right)^{\sigma(E, F)}}=\overline{\mathcal{W}\left(\bigcup_{\gamma \in \Gamma} A_{\gamma}^{\triangleleft}\right)^{\sigma(E, F)}},
$$

and statement (ii) follows.

In the appendix the interested reader will find another very interesting application of this result which shows that an infinite-dimensional version of Farkas' Lemma is true if and only if suitable linear images of positive wedges are weakly closed (cf. Theorem 8.2).

We conclude this preparatory section with a technical lemma which we shall use several times.

Lemma 2.9. Let $(\Omega, \mathscr{F}, \mathbb{P})$ be a probability space and let $Y_{0}, Y$ and $Y_{1}$ be real random variables on $\Omega$ such that $0 \leq Y_{0} \leq Y \leq Y_{1}$ almost surely. Let $f:[0, \infty) \longrightarrow[0, \infty]$ be a convex function. Then for every $a \in[0, \infty]$

$$
\mathbb{E}_{\mathbb{P}}\left[f(Y) \mathbb{1}_{\{Y<a\}}\right] \leq \mathbb{E}_{\mathbb{P}}\left[f\left(Y_{0}\right)\right]+\min \left\{\mathbb{E}_{\mathbb{P}}\left[f\left(Y_{1}\right)\right], f(a)\right\}
$$

Proof. Let $a \in[0, \infty]$ and put

$$
\gamma^{*}:=\inf \{y \geq 0: f \text { is increasing on }(y, \infty)\} .
$$

Obviously, we only have to consider the case $\gamma^{*}<\infty$. In this case, the convex function $f$ is non-increasing on $\left[0, \gamma^{*}\right)$ and increasing on $\left[\gamma^{*}, \infty\right)$. Moreover

$$
f(Y) \mathbb{1}_{\{Y<a\}}=f(Y) \mathbb{1}_{\left\{Y<a \wedge \gamma^{*}\right\}}+f(Y) \mathbb{1}_{\left\{\gamma^{*} \leq Y<a\right\}},
$$

and the claim follows.

\section{The market model}

We now describe in detail the market model. For the necessary background in mathematical finance and stochastic analysis, we refer the reader to the introductory monographs $[20,27]$ and the survey article [12].

Let $\left(\Omega, \mathscr{F},\left(\mathscr{F}_{t}\right)_{t \in[0, T]}, \mathbb{P}\right)$ be a filtered probability space, in which the filtration satisfies the usual conditions of right continuity and completeness, and the time horizon $T$ is assumed to be finite.

We model the discounted price process of $d$ risky assets as an $\mathbb{R}^{d}$-valued semimartingale $S=\left(S_{t}\right)_{t \in[0, T]}$. Let $K$ be an arbitrary wedge in $L^{0}$ consisting of attainable terminal wealths. Note that we are not requiring $K$ to be the wedge of those attainable terminal wealths which arise from admissible trading strategies.

We shall be particularly interested in super-replication for an investor whose preferences are expressed via a utility function. When considering the permissible trading strategies, it is important to take into consideration the investor's wealth preferences. We assume that the investor has a utility function $U:(a, \infty) \rightarrow \mathbb{R}$, 
where $a \in[-\infty, \infty),{ }^{4}$ which is strictly increasing, strictly concave, (continuously) differentiable, and satisfies the Inada conditions

$$
\lim _{x \downarrow a} U^{\prime}(x)=\infty, \quad \lim _{x \uparrow \infty} U^{\prime}(x)=0 .
$$

As usual, we assume that the utility function has Reasonable Asymptotic Elasticity (see [30]). We shall formulate this assumption however in Section 4, in terms of a growth condition on a suitably adapted version of the convex (conjugate) function $\Phi$ (cf. (4.10)).

\section{The separating measures}

Throughout the paper, $K$ always denotes a fixed but arbitrarily chosen wedge in $L^{0}$. Define

$$
\begin{aligned}
M_{1}:= & M_{1}(\mathbb{P} ; K) \\
:= & \left\{\mathbb{Q} \ll \mathbb{P}: \mathbb{Q} \text { is a probability measure, } K \subseteq L^{1}(\mathbb{Q})\right. \text { and } \\
& \left.\mathbb{E}_{\mathbb{Q}}[X] \leq 0 \text { for all } X \in K\right\} .
\end{aligned}
$$

By construction, it follows immediately that

$$
K \subseteq \bigcap_{\mathbb{Q} \in M_{1}} L^{1}(\mathbb{Q}) \subseteq \bigcap_{\mathbb{Q} \in M} L^{1}(\mathbb{Q})
$$

for any non-empty subset $M$ of $M_{1}$. Note that $M_{1}$ consists of probability measures (a fact needed in the proof of Proposition 4.8 and Lemma 5.2). Consider the two convex sets $\mathcal{R}(\mathbb{P}):=\left\{X: X \in L_{+}^{1}(\mathbb{P}), \mathbb{E}_{\mathbb{P}}[X]=1\right\}$ and $\mathcal{A}(\mathbb{P}):=\{\mathbb{Q}: \mathbb{Q}$ is a probability measure and $\mathbb{Q} \ll \mathbb{P}\}$. Then the mapping $R: \mathcal{A}(\mathbb{P}) \rightarrow \mathcal{R}(\mathbb{P}), \mathbb{Q} \mapsto$ $\frac{\mathrm{d} \mathbb{Q}}{\mathrm{d} \mathbb{P}}$ is bijective, and its inverse mapping is given by $L: \mathcal{R}(\mathbb{P}) \rightarrow \mathcal{A}(\mathbb{P}), X \mapsto \mathbb{Q}_{X}$, where $\mathbb{Q}_{X}(A):=\mathbb{E}_{\mathbb{P}}\left[\mathbb{1}_{A} X\right]=\int_{A} X \mathrm{~d} \mathbb{P}$ for any $A \in \mathscr{F}$. Thus, $M_{1} \subseteq \mathcal{A}(\mathbb{P})$ and $R\left(M_{1}\right)=\left\{Y \in L_{+}^{1}(\mathbb{P}): \mathbb{E}_{\mathbb{P}}[Y]=1, X Y \in L^{1}(\mathbb{P})\right.$ and $\mathbb{E}_{\mathbb{P}}[X Y] \leq 0$ for all $\left.X \in K\right\}$.

If it is not mentioned explicitly, then throughout the article, we let $\Phi:[0, \infty) \rightarrow(-\infty, \infty]$ denote an arbitrary convex function, where $\Phi$ may take the value $\infty$ only at $0 .{ }^{5}$ Note that we do not necessarily require $\Phi$ to be the convex conjugate of any utility function.

The set of pricing measures with finite entropy is defined as

$$
\begin{aligned}
M_{\Phi} & :=\left\{\mathbb{Q} \in M_{1}: \Phi\left(\frac{\mathrm{d} \mathbb{Q}}{\mathrm{d} \mathbb{P}}\right) \in L^{1}(\mathbb{P})\right\} \\
& =\left\{\mathbb{Q} \in M_{1}: \mathbb{E}_{\mathbb{P}}\left[\Phi^{+}\left(\frac{\mathrm{d} \mathbb{Q}}{\mathrm{d} \mathbb{P}}\right)\right]<\infty\right\} .
\end{aligned}
$$

By construction, $M_{\Phi}$ depends on the choice of the wedge $K$.

\footnotetext{
${ }^{4}$ We allow $a$ to take any value in $[-\infty, \infty)=\{-\infty\} \cup \mathbb{R}$, but we shall be most interested in the case where $a=-\infty$.

${ }^{5}$ In other words, $\Phi((0, \infty)) \subseteq \mathbb{R}$.
} 
We now state an important growth condition on the convex function $\Phi$, which is related to the condition of Reasonable Asymptotic Elasticity found in [30].

Assumption 4.1. Given any interval $\left[\lambda_{0}, \lambda_{1}\right] \subseteq(0, \infty)$ there exist $\alpha>0$ and $\beta>0$ such that

$$
\Phi^{+}(\lambda y) \leq \alpha \Phi^{+}(y)+\beta(y+1),
$$

for all $y>0$ and all $\lambda \in\left[\lambda_{0}, \lambda_{1}\right]$.

Let $\Phi$ satisfy the growth condition (4.4). Then obviously $\Phi^{+}\left(\lambda \frac{\mathrm{d} \mathbb{Q}}{\mathrm{d} \mathbb{P}}\right) \in L^{1}(\mathbb{P})$ for any $\lambda>0$ and any $\mathbb{Q} \in M_{\Phi}$.

Primarily, we are interested in the case where $\Phi$ is the convex conjugate of a utility function $U$, i. e., the Legendre conjugate of the convex function $-U(-\cdot)$ : $(-\infty,-a) \longrightarrow \mathbb{R}($ cf. $[28, \S 26]):$

$$
\Phi(y):=\sup _{x>a}\{U(x)-x y\}, \quad y \geq 0 .
$$

In this case, the growth condition (4.4) and the Reasonable Asymptotic Elasticity of $U$, introduced by Schachermayer, are equivalent (cf. [16]).

It is well-known that under the Inada conditions (3.1) the convex conjugate $\Phi$ is strictly convex and continuously differentiable on $(0, \infty)$, satisfying

$$
\lim _{y \rightarrow \infty} \Phi(y)=\lim _{x \rightarrow a} U(x) \quad \text { and } \quad \Phi^{\prime}=-I,
$$

where $I:(0, \infty) \longrightarrow(a, \infty)$ denotes the inverse function of $U^{\prime}$. Moreover,

$$
\Phi(y)=U(I(y))-y I(y)
$$

for all $y>0($ cf. $[28, \S 26])$.

Throughout the article we shall assume the following

Assumption 4.2. $M_{\Phi} \neq \emptyset$.

Remark 4.3. Assumption 4.2 rules out "utility-based arbitrage strategies" (see [5, Section 1.2]), but not necessarily a free lunch with vanishing risk. As our analysis shows, it is not necessary to assume the stronger condition

$$
\left\{\mathbb{Q} \in M_{\Phi}: \mathbb{Q} \sim \mathbb{P}\right\} \neq \emptyset
$$

which was required throughout in [5].

Definition 4.4. We say that a measure $\mathbb{Q} \ll \mathbb{P}$ has finite loss-entropy if

$$
\mathbb{E}_{\mathbb{P}}\left[\Phi^{+}\left(\frac{\mathrm{d} \mathbb{Q}}{\mathrm{d} \mathbb{P}}\right) \mathbb{1}_{\left\{\frac{\mathrm{d} \mathbb{Q}}{\mathrm{d}} \geq 1\right\}}\right]<\infty .
$$

The set of pricing measures with finite loss-entropy is therefore given by

$$
\widehat{M}_{\Phi}:=\left\{\mathbb{Q} \in M_{1}: \mathbb{E}_{\mathbb{P}}\left[\Phi^{+}\left(\frac{\mathrm{d} \mathbb{Q}}{\mathrm{d} \mathbb{P}}\right) \mathbb{1}_{\left\{\frac{\mathrm{d}}{\mathrm{dP}} \geq 1\right\}}\right]<\infty\right\} .
$$


Clearly, $M_{\Phi} \subseteq \widehat{M}_{\Phi}$. Since

$$
0 \leq \Phi^{+}\left(\frac{\mathrm{d} \mathbb{Q}}{\mathrm{dP}}\right) \mathbb{1}_{\left\{a \leq \frac{\mathrm{d} \mathbb{Q}}{\mathrm{dP}}<b\right\}} \leq \max \left\{\Phi^{+}(a), \Phi^{+}(b)\right\}<\infty
$$

for any $\mathbb{Q} \ll \mathbb{P}$ and $0<a<b$, the choice of the constant 1 in equation (4.8) is arbitrary; we could actually choose any positive number, and the set in (4.9) would not change. We use the terminology "loss-entropy" because for events with large $\frac{\mathrm{d} \mathbb{Q}}{\mathrm{dP}}$, an inspection of equation (4.6) shows that $\Phi\left(\frac{\mathrm{d} \mathbb{Q}}{\mathrm{dP}}\right)$ is related to the value of the utility function $U(x)$ where $x$ is close to the critical wealth $a$. Typically, pricing measures $\mathbb{Q} \in M_{1}$ give large probabilities (relative to the real world measure $\mathbb{P}$ ) to large negative asset prices.

We now define a modification $\widehat{\Phi}$ of $\Phi$ which is finite at 0 , but remains convex and satisfies $\widehat{\Phi} \leq \Phi$. Since the left-sided derivative $l^{*}:=\left(D^{-} \Phi\right)(1)$ of the convex function $\Phi$ at $y=1$ always exists, it follows that

$$
\widehat{\Phi}(y):= \begin{cases}\Phi(y), & \text { if } y \geq 1 \\ \Phi(1)+l^{*}(y-1) & \text { if } 0 \leq y<1\end{cases}
$$

defines a function $\widehat{\Phi}$ on $[0, \infty)$ with values in $\mathbb{R}$. If $\Phi$ satisfies the growth condition (4.4), so does $\widehat{\Phi}$. Moreover,

$$
\widehat{M}_{\Phi}=M_{\widehat{\Phi}}
$$

Remark 4.5. (i) Since $\Phi$ and $\widehat{\Phi}$ are convex, it follows easily that $M_{\Phi}$ and $\widehat{M}_{\Phi}$ are convex.

(ii) If $\lim _{y \rightarrow \infty}\left(D^{-} \Phi\right)(y)<\infty$ (i.e., $\Phi$ is asymptotically linear as $y \rightarrow \infty$ ), then $\widehat{M}_{\Phi}=M_{1}$, because then $\widehat{\Phi}$ can be bounded above by a linear function (see also Remark 6.6).

(iii) If $\Phi(0)<\infty$, then $\widehat{M}_{\Phi}=M_{\Phi}$, because in this case $0 \leq \Phi^{+}\left(\frac{\mathrm{d} \mathbb{Q}}{\mathrm{d} \mathbb{P}}\right) \mathbb{1}_{\left\{0 \leq \frac{\mathrm{d} \mathbb{Q}}{\mathrm{dP}}<1\right\}} \leq$ $\max \left\{\Phi^{+}(0), \Phi^{+}(1)\right\}<\infty$ for any $\mathbb{Q} \ll \mathbb{P}$.

(iv) The introduction of the sets $\widehat{M}_{\Phi}$ and $M_{\widehat{\Phi}}$ here is original. We note that the latter set is related to the conjugate function of the Young function $\widehat{U}$ associated to the utility function $U$, via the transformation $\widehat{U}(x):=-U(-|x|)+U(0)$ (cf. [7]). In this way, $M_{\widehat{\Phi}}$ shows up very naturally.

Let us recall that a convex subset $F$ of a given convex set $C$ is called a face of $C$ if $\alpha x+(1-\alpha) y \in F$ with $x, y \in C$ and $0<\alpha<1$ imply $x, y \in F$ (cf. [2]). By using similar estimation techniques as inserted in the respective proofs of Proposition 18 in [5] and Theorem 4 in [6], we reveal a significant geometric fact which will become central to our further analysis (cf. Theorem 6.4):

Lemma 4.6. If $\Phi$ satisfies the growth condition (4.4) then the following statements hold:

(i) $\widehat{M}_{\Phi}$ is a face in $M_{1}$.

(ii) Let $\mathbb{Q}_{0}, \mathbb{Q}_{1} \in M_{1}, 0<x<1$, and assume that $\mathbb{Q}:=x \mathbb{Q}_{0}+(1-x) \mathbb{Q}_{1} \in \widehat{M}_{\Phi}$. If $\mathbb{Q}_{0} \in M_{\Phi}$ or $\mathbb{Q}_{1} \in M_{\Phi}$ then $\mathbb{Q} \in M_{\Phi}$. 
Proof. Let $\mathbb{Q}_{0}, \mathbb{Q}_{1} \in M_{1}, 0<x<1$ and define $\mathbb{Q}:=x \mathbb{Q}_{0}+(1-x) \mathbb{Q}_{1}$. To prove statement (i), note that since $\widehat{M}_{\Phi}$ is convex, we only have to show that $\mathbb{Q} \in \widehat{M}_{\Phi}$ implies that $\mathbb{Q}_{0}, \mathbb{Q}_{1} \in \widehat{M}_{\Phi}$. Suppose that $\mathbb{Q} \in \widehat{M}_{\Phi}=M_{\widehat{\Phi}}$. Since $0 \leq \frac{\mathrm{d} \mathbb{Q}_{0}}{\mathrm{~d} \mathbb{P}}=$ $\frac{1}{x} \frac{\mathrm{d} \mathbb{Q}}{\mathrm{d} \mathbb{P}}-\frac{(1-x)}{x} \frac{\mathrm{d} \mathbb{Q}_{1}}{\mathrm{~d} \mathbb{P}} \leq \frac{1}{x} \frac{\mathrm{d} \mathbb{Q}}{\mathrm{d} \mathbb{P}}-$ a.s., Lemma 2.9 and the growth condition (4.4) of $\widehat{\Phi}$ imply that

$$
\mathbb{E}_{\mathbb{P}}\left[\widehat{\Phi}^{+}\left(\frac{\mathrm{d} \mathbb{Q}_{0}}{\mathrm{~d} \mathbb{P}}\right)\right] \leq \widehat{\Phi}^{+}(0)+\mathbb{E}_{\mathbb{P}}\left[\widehat{\Phi}^{+}\left(\frac{1}{x} \frac{\mathrm{d} \mathbb{Q}}{\mathrm{dP}}\right)\right]<\infty .
$$

Hence, $\mathbb{Q}_{0} \in \widehat{M}_{\Phi}$. Similarly, $\mathbb{Q}_{1} \in \widehat{M}_{\Phi}$.

To prove statement (ii), we may suppose without loss of generality that $\mathbb{Q}_{0} \in M_{\Phi}$. Since $x \frac{\mathrm{d} \mathbb{Q}_{0}}{\mathrm{~d} \mathbb{P}} \leq \frac{\mathrm{d} \mathbb{Q}}{\mathrm{d} \mathbb{P}} \mathbb{P}$-a.s., Lemma 2.9 and the growth condition (4.4) of $\Phi$ imply that

$$
\mathbb{E}_{\mathbb{P}}\left[\Phi^{+}\left(\frac{\mathrm{d} \mathbb{Q}}{\mathrm{d} \mathbb{P}}\right) \mathbb{1}_{\left\{\frac{\mathrm{d}}{\mathrm{d} \mathbb{P}}<1\right\}}\right] \leq \mathbb{E}_{\mathbb{P}}\left[\Phi^{+}\left(x \frac{\mathrm{d} \mathbb{Q}_{0}}{\mathrm{dP}}\right)\right]+\Phi^{+}(1)<\infty .
$$

Since $\mathbb{Q} \in \widehat{M}_{\Phi}$, it therefore follows that $\mathbb{Q} \in M_{\Phi}$.

We now include a general representation result which holds for arbitrary nonempty faces of convex sets, consisting of probability measures (such as it is the case for $M_{1}$ ).

Proposition 4.7. Let $M$ be a face of $M_{1}$. If $M \neq \emptyset$ then

$$
[M] \cap M_{1}=M .
$$

Proof. Clearly, $\emptyset \neq M \subseteq[M] \cap M_{1}$. Let $\mathbb{Q} \in[M] \cap M_{1}$. First, due to (2.2), it follows that

$$
[M]=\mathcal{W}(M)-\mathcal{W}(M) .
$$

Therefore, since $\mathbb{Q}$ in particular is a probability measure, $\mathbb{Q}$ can be written as $\mathbb{Q}=(1+\beta) \mathbb{Q}_{1}-\beta \mathbb{Q}_{0}$ where $\mathbb{Q}_{0}, \mathbb{Q}_{1} \in M \subseteq M_{1}$ and $\beta \geq 0$. Thus $\mathbb{Q}_{1}=\frac{1}{1+\beta} \mathbb{Q}_{+}+\frac{\beta}{1+\beta} \mathbb{Q}_{0}$, and the assumption implies that $\mathbb{Q} \in M$.

Proposition 4.8. If $\Phi$ satisfies the growth condition (4.4) and $M_{\Phi} \neq \emptyset$ then

$$
\widehat{M}_{\Phi}=\left[M_{\Phi}\right] \cap M_{1}
$$

Proof. Firstly, due to Lemma 4.6 and Proposition 4.7, we have

$$
\left[M_{\Phi}\right] \cap M_{1} \subseteq\left[\widehat{M}_{\Phi}\right] \cap M_{1}=\widehat{M}_{\Phi}
$$

Let $\mathbb{Q}_{1} \in \widehat{M}_{\Phi}$. Choose any $\mathbb{Q}_{0} \in M_{\Phi}$ and define $\mathbb{Q}:=\frac{1}{2} \mathbb{Q}_{0}+\frac{1}{2} \mathbb{Q}_{1}$. From Lemma 4.6 we see that $\mathbb{Q} \in M_{\Phi}$. Hence, $\mathbb{Q}_{1}=2 \mathbb{Q}-\mathbb{Q}_{0} \in\left[M_{\Phi}\right]$. 


\section{Main results: a general approach by duality}

In the following, we extend the model approach of [5], which does not only allow us to substitute the wedge $K^{\mathrm{adm}}$ of admissible strategies by more general candidates including the wedge of acceptable strategies (cf. [18]), or the wedge of permissible strategies (cf. [26]). This extension also enables to replace the set $M_{\Phi}$ by other suitable non-empty subsets of $M_{1}$ (cf. Corollary 5.5) and reveals the geometry of a suitable class of wedges which appears naturally in the analysis of super-replication prices for unbounded contingent claims.

To this end, let $K$ be a fixed wedge in $L^{0}$ and $M$ be a fixed non-empty subset of $M_{1} \equiv M_{1}(\mathbb{P} ; K) \subseteq \mathcal{A}(\mathbb{P})$. As in [5], we consider the pair of vector spaces

$$
E:=\bigcap_{\mathbb{Q} \in M} L^{1}(\mathbb{Q}) \quad \text { and } \quad F:=[R(M)]=\left[\frac{\mathrm{d} \mathbb{Q}}{\mathrm{dP}}: \mathbb{Q} \in M\right] \subseteq L^{1}(\mathbb{P}),
$$

where $R(\mathbb{Q})=\frac{\mathrm{d} \mathbb{Q}}{\mathrm{dP}}$ for all $\mathbb{Q} \in \mathcal{A}(\mathbb{P})$ (cf. Section 4). Obviously, if $z \in E$ and $w \in F \subseteq L^{1}(\mathbb{P})$ then $z w \in L^{1}(\mathbb{P})$. Thus the bilinear form $\langle\cdot, \cdot\rangle:(E, F) \rightarrow \mathbb{R}$ defined by

$$
\langle z, w\rangle:=\mathbb{E}_{\mathbb{P}}[z w]
$$

is well defined, making $(E, F)$ a bilinear system. From now on, all polar wedges are defined with respect to this bilinear system. Therefore, their structure depends on the choice of the wedge $K$ and the set $M$. Note that $E$ is a vector sublattice of $L^{0}$ which contains the wedge $K$ (cf. (4.1)).

A standard measure theoretic argument shows that the linear functionals $E \ni z \mapsto \mathbb{E}_{\mathbb{P}}[z w]$ are non-degenerate for each $w \in F \backslash\{0\}$. Hence, the bilinear system $(E, F)$ is a left dual system. Consequently, $F$ is the topological dual of $E$ under the weak topology $\sigma(E, F)$ :

$$
F=(E, \sigma(E, F))^{\prime}
$$

Let $X \in E$ be an arbitrary contingent claim and $C \subseteq E$ be an arbitrary wedge which contains the (fixed) wedge $K$. Recall that

$$
s_{E}(C)=\bigcap_{\mathbb{Q} \in M}\left(C-L_{+}^{1}(\mathbb{Q})\right)=C-E_{+} \subseteq E
$$

describes the umbrella hull of $C$ (cf. Proposition 2.3). Consider the set

$$
A_{X}(C):=\{x \in \mathbb{R}: X \leq x+G \text { for some } G \in C\}
$$

and assume that $A_{X}(K) \neq \emptyset$. Then $A_{X}(K)$ is bounded from below, and we have

$$
-\infty<\sup _{\mathbb{Q} \in M} \mathbb{E}_{\mathbb{Q}}[X] \leq \inf \left(A_{X}(K)\right)<+\infty .
$$


Obviously, $A_{X}(K) \subseteq A_{X}(C)$. If $A_{X}(C)$ is bounded from below, we put

$$
\bar{\pi}(X ; C):=\inf \left(A_{X}(C)\right) .
$$

Note that $\bar{\pi}(X ; C) \leq \bar{\pi}(X ; K)$.

Definition 5.1. Let $M$ be a non-empty subset of $M_{1}=M_{1}(\mathbb{P} ; K)$ and $C \subseteq E:=$ $\bigcap_{\mathbb{Q} \in M} L^{1}(\mathbb{Q})$ be a wedge which contains the wedge $K$. Let $X \in E$. If $A_{X}(C)$ is non-empty and bounded from below then the real number

$$
\bar{\pi}(X ; C):=\inf \left(A_{X}(C)\right)=\inf \{x \in \mathbb{R}: X \leq x+G \text { for some } G \in C\}
$$

is called $M$-based super-replication price of $X$.

Due to Proposition 2.3, it follows immediately that

$\bar{\pi}(X ; C)=\inf \left\{x \in \mathbb{R}: X-x \in s_{E}(C)\right\}=\inf \left\{x \in \mathbb{R}: X-x \in \bigcap_{\mathbb{Q} \in M}\left(C-L_{+}^{1}(\mathbb{Q})\right)\right\}$.

Hence, $\bar{\pi}(X ; C)=\bar{\pi}\left(X ; s_{E}(C)\right)$. If $M:=M_{\Phi}$, where $\Phi$ is the convex conjugate of a utility function, we call $\bar{\pi}\left(X ; C_{\Phi}\right)$ a utility-based super-replication price of $X$ (cf. (6.2)). Note that in the admissible case our utility-based super-replication price of $X$ coincides with the weak super-replication price of [5].

The following important result reveals that the dual wedge of the umbrella wedge $s_{E}(K)$ is contained in the positive wedge of $L^{1}(\mathbb{P})$ and can be represented with the help of suitable probability measures. It is not only crucial for our analysis in this paper. By choosing the "right" dual pair namely, it opens up a door to a canonical duality and a general representation of $M$-based super-replication prices (cf. [25]).

Lemma 5.2. Let $K$ be an arbitrary wedge in $L^{0}$, and let $\emptyset \neq M \subseteq M_{1}=M_{1}(\mathbb{P} ; K)$. Then

$$
F \cap \mathcal{W}\left(R\left(M_{1}\right)\right)=s_{E}(K)^{\triangleleft}=\left(-L_{+}^{\infty}(\mathbb{P})\right)^{\triangleleft} \cap K^{\triangleleft}=L_{+}^{1}(\mathbb{P}) \cap K^{\triangleleft} .
$$

In particular, the set $F \cap \mathcal{W}\left(R\left(M_{1}\right)\right)$ is $\sigma(F, E)$-closed, and it contains $\overline{\mathcal{W}}(R(M))^{\sigma(F, E)}$

Proof. First, we show that

$$
F \cap \mathcal{W}\left(R\left(M_{1}\right)\right) \subseteq s_{E}(K)^{\triangleleft} \subseteq\left(-L_{+}^{\infty}(\mathbb{P})\right)^{\triangleleft} \cap K^{\triangleleft}=L_{+}^{1}(\mathbb{P}) \cap K^{\triangleleft} .
$$

To this end, let $\mathbb{Q} \in M_{1}$ such that $R(\mathbb{Q})=\frac{\mathrm{d} \mathbb{Q}}{\mathrm{d} \mathbb{P}} \in F$ and $X \in s_{E}(K)$. Then there exists $Y \in K \subseteq L^{1}(\mathbb{Q})$ such that $X \leq Y$ and

$$
\left\langle X, \frac{\mathrm{d} \mathbb{Q}}{\mathrm{d} \mathbb{P}}\right\rangle=\mathbb{E}_{\mathbb{Q}}[X] \leq \mathbb{E}_{\mathbb{Q}}[Y] \leq 0
$$

Thus, $F \cap R\left(M_{1}\right) \subseteq s_{E}(K)^{\triangleleft}$. Since $\emptyset \neq R(M) \subseteq F \cap R\left(M_{1}\right)$, we may apply Lemma 2.1 to the wedge $F$, and it consequently follows that

$$
F \cap \mathcal{W}\left(R\left(M_{1}\right)\right)=\mathcal{W}\left(F \cap R\left(M_{1}\right)\right) \subseteq s_{E}(K)^{\triangleleft} .
$$


Since $L^{\infty}(\mathbb{P}) \subseteq L^{1}(\mathbb{Q})$ for any $\mathbb{Q} \in M_{1}$, we have $L^{\infty}(\mathbb{P}) \subseteq E$ and hence

$$
-L_{+}^{\infty}(\mathbb{P}) \cup K \subseteq K-E_{+}=s_{E}(K) \subseteq E .
$$

Since

$$
\begin{aligned}
\left(-L_{+}^{\infty}(\mathbb{P})\right)^{\triangleleft} & =F \cap\left\{W \in L^{1}(\mathbb{P}): \mathbb{E}_{\mathbb{P}}[W V] \geq 0 \text { for all } V \in L_{+}^{\infty}(\mathbb{P})\right\} \\
& \subseteq F \cap\left\{W \in L^{1}(\mathbb{P}): \mathbb{E}_{\mathbb{P}}\left[W \mathbb{1}_{\{W<0\}}\right] \geq 0\right\} \\
& =F \cap\left\{W \in L^{1}(\mathbb{P}): \mathbb{E}_{\mathbb{P}}\left[W^{-}\right]=0\right\} \\
& =F \cap L_{+}^{1}(\mathbb{P}) \\
& \subseteq\left(-L_{+}^{\infty}(\mathbb{P})\right)^{\triangleleft},
\end{aligned}
$$

Lemma 2.8 and inclusion (5.5) imply that

$$
s_{E}(K)^{\triangleleft} \subseteq\left(-L_{+}^{\infty}(\mathbb{P})\right)^{\triangleleft} \cap K^{\triangleleft}=L_{+}^{1}(\mathbb{P}) \cap K^{\triangleleft} .
$$

Hence,

$$
F \cap \mathcal{W}\left(R\left(M_{1}\right)\right) \subseteq s_{E}(K)^{\triangleleft} \subseteq\left(-L_{+}^{\infty}(\mathbb{P})\right)^{\triangleleft} \cap K^{\triangleleft}=L_{+}^{1}(\mathbb{P}) \cap K^{\triangleleft} .
$$

It remains to prove that $L_{+}^{1}(\mathbb{P}) \cap K^{\triangleleft} \subseteq F \cap \mathcal{W}\left(R\left(M_{1}\right)\right)$. To this end, let $Y \in L_{+}^{1}(\mathbb{P}) \cap K^{\triangleleft}=L_{+}^{1}(\mathbb{P}) \cap K^{\triangleleft} \cap F$. Then $0 \leq Y=\sum_{k=1}^{n} \alpha_{k} \frac{\mathrm{d} \mathbb{Q}_{k}}{\mathrm{~d} \mathbb{P}}$, for some $n \in \mathbb{N}, \alpha_{1}, \ldots, \alpha_{n} \in \mathbb{R}$ and $\mathbb{Q}_{1}, \ldots, \mathbb{Q}_{n} \in M \subseteq M_{1}$, and $\mathbb{E}_{\mathbb{P}}[X Y] \leq 0$ for all $X \in K$. Consider $\mu:=\sum_{k=1}^{n} \alpha_{k} \mathbb{Q}_{k}$. Since each $\mathbb{Q}_{k}$ in particular is a probability measure, it follows that

$$
0 \leq \mathbb{E}_{\mathbb{P}}[Y]=\sum_{k=1}^{n} \alpha_{k}=\mu(\Omega) .
$$

If $\mu(\Omega)=\mathbb{E}_{\mathbb{P}}[Y]=0$ then $Y=0 \in F \cap \mathcal{W}\left(R\left(M_{1}\right)\right)$. If $\mu(\Omega)>0$, it follows that $\mathbb{Q}:=\sum_{k=1}^{n} \frac{\alpha_{k}}{\mu(\Omega)} \mathbb{Q}_{k}$ is a probability measure, $\mathbb{Q} \ll \mathbb{P}$ and

$$
\mu(\Omega) \mathbb{E}_{\mathbb{Q}}[X]=\mathbb{E}_{\mathbb{P}}[X Y] \leq 0
$$

for all $X \in K$. Consequently, $\mathbb{Q} \in M_{1}$, and we obtain

$$
Y=\sum_{k=1}^{n} \alpha_{k} \frac{\mathrm{d} \mathbb{Q}_{k}}{\mathrm{~d} \mathbb{P}}=\mu(\Omega) \frac{\mathrm{d} \mathbb{Q}}{\mathrm{d} \mathbb{P}}=\mu(\Omega) R(\mathbb{Q}) \in F \cap \mathcal{W}\left(R\left(M_{1}\right)\right) .
$$

Theorem 5.3. Let $K$ be an arbitrary wedge in $L^{0}$ and let $\emptyset \neq M \subseteq M_{1}=M_{1}(\mathbb{P} ; K)$. Put $c_{E}(K):={\overline{s_{E}(K)}}^{\sigma(E, F)}$. Then the following statements are equivalent:

(i) $\overline{\mathcal{W}(R(M))}^{\sigma(F, E)}=F \cap \mathcal{W}\left(R\left(M_{1}\right)\right)$;

(ii) $R(M)^{\triangleleft}=c_{E}(K)$;

(iii) $\overline{\mathcal{W}(R(M))}^{\sigma(F, E)}=\left(c_{E}(K)\right)^{\triangleleft}=s_{E}(K)^{\triangleleft}$. 
Proof. Assume that (i) holds. Then Proposition 2.7 and Lemma 5.2 both together imply that

$$
\begin{aligned}
R(M)^{\triangleleft} & \left.=\left(R(M)^{\triangleleft \triangleleft}\right)^{\triangleleft}=(\overline{\mathcal{W}(R(M))})^{\sigma(F, E)}\right)^{\triangleleft} \\
& \stackrel{(i)}{=}\left(F \cap \mathcal{W}\left(R\left(M_{1}\right)\right)\right)^{\triangleleft}=\left(s_{E}(K)^{\triangleleft}\right)^{\triangleleft}=c_{E}(K),
\end{aligned}
$$

and (ii) follows.

Now assume that (ii) holds. Again, we may apply Proposition 2.7 and Lemma 5.2 and obtain

$$
\overline{\mathcal{W}}(R(M))^{\sigma(F, E)}=R(M)^{\triangleleft \triangleleft} \stackrel{(i i)}{=} c_{E}(K)^{\triangleleft}=\left(s_{E}(K)^{\triangleleft \triangleleft}\right)^{\triangleleft}=s_{E}(K)^{\triangleleft} .
$$

Hence, statement (iii) follows.

If (iii) is true, Lemma 5.2 immediately implies (i).

A natural question is to ask for suitable candidates $M$ which satisfy condition (ii) and hence the equivalent relations (i) respectively (iii) of Theorem 5.3. In fact, we shall recognise again that one candidate of this type is given by $\widehat{M}_{\Phi}$ (cf. [25]).

Theorem 5.4. Let $K$ be an arbitrary wedge in $L^{0}$, and let $\emptyset \neq M$ be a face of $M_{1}=M_{1}(\mathbb{P} ; K)$. Then

$$
R(M)^{\triangleleft}=c_{E}(K) .
$$

Proof. Firstly, due to Proposition 4.7 we have

$$
R(M)=R\left([M] \cap M_{1}\right)=F \cap R\left(M_{1}\right),
$$

so that

$$
\mathcal{W}(R(M))=F \cap \mathcal{W}\left(R\left(M_{1}\right)\right)
$$

(by Lemma 2.1). Consequently, Lemma 5.2 implies

$$
\mathcal{W}(R(M))^{\triangleleft}=\left(s_{E}(K)^{\triangleleft}\right)^{\triangleleft}=s_{E}(K)^{\triangleleft \triangleleft} .
$$

Now, we may apply Proposition 2.6 and Proposition 2.7, and it consequently follows that

$$
R(M)^{\triangleleft}=\mathcal{W}(R(M))^{\triangleleft}=s_{E}(K)^{\triangleleft \triangleleft}={\overline{s_{E}(K)}}^{\sigma(E, F)}=c_{E}(K) .
$$

Corollary 5.5. Let $K$ be an arbitrary wedge in $L^{0}$, and let $\emptyset \neq M \subseteq M_{1}=M_{1}(\mathbb{P}, K)$ such that

$$
\overline{\mathcal{W}}(R(M))^{\sigma(F, E)}=F \cap \mathcal{W}\left(R\left(M_{1}\right)\right),
$$

respectively

$$
R(M)^{\triangleleft}=c_{E}(K) .
$$

Let $X \in E$. Then the set $A_{X}\left(c_{E}(K)\right)$ is bounded from below and

$$
\bar{\pi}\left(X ; c_{E}(K)\right)=\sup _{\mathbb{Q} \in M} \mathbb{E}_{\mathbb{Q}}[X] .
$$


Proof. Since obviously

$$
c_{E}(K)-E_{+}={\overline{s_{E}(K)}}^{\sigma(E, F)}-E_{+} \subseteq{\overline{K-E_{+}}}^{\sigma(E, F)}=c_{E}(K),
$$

$c_{E}(K)$ is an umbrella wedge in E. Hence, Theorem 5.3 implies that

$$
\begin{aligned}
\bar{\pi}\left(X ; c_{E}(K)\right) & =\inf \left\{x \in \mathbb{R}: X-x \in c_{E}(K)\right\} \\
& =\inf \left\{x \in \mathbb{R}: X-x \in R(M)^{\triangleleft}\right\} \\
& =\inf \left\{x \in \mathbb{R}: \mathbb{E}_{\mathbb{Q}}[X-x] \leq 0 \forall \mathbb{Q} \in M\right\} \\
& =\inf \left\{x \in \mathbb{R}: \mathbb{E}_{\mathbb{Q}}[X] \leq x \forall \mathbb{Q} \in M\right\} \\
& =\sup _{\mathbb{Q} \in M} \mathbb{E}_{\mathbb{Q}}[X],
\end{aligned}
$$

and the claim follows.

Corollary 5.6. Let $K$ be an arbitrary wedge in $L^{0}$. If $\Phi$ satisfies the growth condition (4.4) and $\widehat{M}_{\Phi} \neq \emptyset$ then

$$
R\left(\widehat{M}_{\Phi}\right)^{\triangleleft}=c_{\widehat{E_{\Phi}}}(K)
$$

where the polarisation $\triangleleft$ is based on the bilinear system $\left(\widehat{E_{\Phi}}, \widehat{F_{\Phi}}\right)$ which is given by $\widehat{E_{\Phi}}=\bigcap_{\mathbb{Q} \in \widehat{M}_{\Phi}} L^{1}(\mathbb{Q})$ and $\widehat{F_{\Phi}}=\left[R\left(\widehat{M}_{\Phi}\right)\right]$.

Proof. The result immediately follows from Lemma 4.6 and Theorem 5.4, applied to $M:=\widehat{M}_{\Phi}$ and the related bilinear system $(E, F):=\left(\widehat{E_{\Phi}}, \widehat{F_{\Phi}}\right)(\operatorname{cf} .5 .1)$.

Note that Lemma 5.2 and Theorem 5.3 hold for any non-empty subset $M$ of $M_{1}$. In particular, we may apply them to $M \in\left\{M_{\Phi}, \widehat{M_{\Phi}}\right\}$ - presupposed that $M_{\Phi} \neq \emptyset$ respectively $\widehat{M_{\Phi}} \neq \emptyset$. The case $M=\widehat{M_{\Phi}}$ is discussed in detail in [25]. We now apply our general results to the case $M=M_{\Phi}$ and provide a well-known representation of the umbrella wedge $c_{E}(K)$ in Theorem 6.4 , implying a generalisation of Theorem 5 in [5].

\section{The special case $M=M_{\Phi}$}

As before, we fix an arbitrary wedge $K$ in $L^{0}$. Assume that $\Phi$ satisfies the growth condition $(4.4)$ and $M_{\Phi} \neq \emptyset$. Let $E_{\Phi}:=\bigcap_{\mathbb{Q} \in M_{\Phi}} L^{1}(\mathbb{Q})$ be the vector space of all $M_{\Phi}$-integrable contingent claims. Recall that $K \subseteq \bigcap_{\mathbb{Q} \in M_{1}} L^{1}(\mathbb{Q}) \subseteq E_{\Phi}$. Define the wedge

$$
K_{\Phi}:=s_{E_{\Phi}}(K)=K-\left(E_{\Phi}\right)_{+}=\bigcap_{\mathbb{Q} \in M_{\Phi}}\left(K-L_{+}^{1}(\mathbb{Q})\right)
$$

of all $M_{\Phi}$-integrable contingent claims that can be dominated by a terminal wealth in $K$. We now consider the pair of vector spaces

$$
E_{\Phi}:=\bigcap_{\mathbb{Q} \in M_{\Phi}} L^{1}(\mathbb{Q}) \quad \text { and } \quad F_{\Phi}:=\left[R\left(M_{\Phi}\right)\right] .
$$


It is well-known that the wedge $K^{\text {adm }}$ of terminal wealths arising from zero-financed admissible trading strategies is not large enough for the purposes of a duality theory when considering unbounded wealth. Similarly, in our general setting the wedge $K_{\Phi}$ may not be large enough in order to obtain a dual relationship of type (1.4). Along the lines of equation (1.5) we therefore define the larger wedge

$$
C_{\Phi}:=\bigcap_{\mathbb{Q} \in M_{\Phi}}{\overline{K-L_{+}^{1}(\mathbb{Q})}}^{L^{1}(\mathbb{Q})},
$$

which in fact will turn out to be the closure of $K_{\Phi}$ with respect to the weak topology $\sigma\left(E_{\Phi}, F_{\Phi}\right)$ (see Theorem 6.4 ). Both, $K_{\Phi}$ and $C_{\Phi}$ are umbrella wedges in $E_{\Phi}$, and

$$
K \subseteq K_{\Phi} \subseteq C_{\Phi} \subseteq E_{\Phi}
$$

We interpret $C_{\Phi}$ as the wedge of contingent claims which can be approximated by super-replicable claims, where the investor has only utility-induced restrictions on wealth.

The following result shows that the wedges $C_{\widehat{\Phi}}$ and $C_{\Phi}$ are identical (c.f. $(4.10))$.

Lemma 6.1. If $\Phi$ satisfies the growth condition (4.4) and $M_{\Phi} \neq \emptyset$ then $C_{\widehat{\Phi}}=C_{\Phi}$.

Proof. Since $M_{\Phi} \subseteq \widehat{M}_{\Phi}=M_{\widehat{\Phi}}$ it follows that $C_{\widehat{\Phi}} \subseteq C_{\Phi}$. Therefore it suffices to show that for any $\mathbb{Q}_{1} \in \widehat{M}_{\Phi}$ there exists $\mathbb{Q} \in M_{\Phi}$ such that $\overline{K-L_{+}^{1}(\mathbb{Q})}{ }^{L^{1}(\mathbb{Q})} \subseteq$ ${\overline{K-L_{+}^{1}\left(\mathbb{Q}_{1}\right)}}^{L^{1}\left(\mathbb{Q}_{1}\right)}$. Indeed, let $\mathbb{Q}_{1} \in \widehat{M}_{\Phi}$, take any $\mathbb{Q}_{0} \in M_{\Phi}$ and define $\mathbb{Q}=\frac{1}{2} \mathbb{Q}_{0}+\frac{1}{2} \mathbb{Q}_{1}$. Then $\mathbb{Q} \in M_{\Phi}$ (due to Lemma 4.6). If $X \in{\overline{K-L_{+}^{1}(\mathbb{Q})}}^{L^{1}(\mathbb{Q})}$ then there exists $\tilde{X}_{n} \in K$ and $R_{n} \in L_{+}^{1}(\mathbb{Q})$ such that $X_{n}:=\widetilde{X}_{n}-R_{n} \stackrel{L^{1}(\mathbb{Q})}{\longrightarrow} X$. Since $\left\|R_{n}\right\|_{L^{1}\left(\mathbb{Q}_{1}\right)} \leq 2\left\|R_{n}\right\|_{L^{1}(\mathbb{Q})}$ we have $X_{n} \in K-L_{+}^{1}\left(\mathbb{Q}_{1}\right)$. Moreover, $\left\|X-X_{n}\right\|_{L^{1}\left(\mathbb{Q}_{1}\right)} \leq 2$ $\left\|X-X_{n}\right\|_{L^{1}(\mathbb{Q})} \rightarrow 0$ as $n \rightarrow \infty$.

Remark 6.2. Lemma 6.1 reveals an interesting economic insight into the wedge $C_{\Phi}$. On inspection of definition (6.2), one is lead to believe that $C_{\Phi}$ is highly dependent on $\Phi$, and therefore on $U$. However, as a result of Lemma 6.1, we can replace in this definition the set $M_{\Phi}$ by the set $M_{\widehat{\Phi}}=\widehat{M}_{\Phi}$ of pricing measures with finite loss-entropy.

The definition of the loss-entropy of a pricing measure only depends upon the conjugate function $\Phi(y)$ for arbitrarily large values of $y$ (see the discussion after equation (4.9)). In turn, the behaviour of $\Phi(y)$ for large values of $y$ corresponds to the behaviour of the utility function $U(x)$ for large negative values of $x$. Therefore, although the trader is restricted in their choice of terminal wealths by their utility function, this restriction actually depends only upon the investor's preferences towards asymptotically large losses. 
The following statement is a slight extension of a fundamental result of Biagini and Frittelli (cf. [6], Theorem 4). However, it shows again that we are allowed to replace the smaller set $M_{\Phi}$ by the larger set $\widehat{M}_{\Phi}$.

Proposition 6.3. If $\Phi$ satisfies the growth condition (4.4) and $M_{\Phi} \neq \emptyset$ then

$$
R\left(M_{\Phi}\right)^{\triangleleft}=C_{\Phi}=R\left(\widehat{M}_{\Phi}\right)^{\triangleleft}
$$

where the polarisation $\triangleleft$ is based on the bilinear system $\left(E_{\Phi}, F_{\Phi}\right)$. In particular, $C_{\Phi}$ is $\sigma\left(E_{\Phi}, F_{\Phi}\right)$-closed.

Proof. Due to Theorem 4 in [6], we only have to show the inclusion $C_{\Phi} \subseteq R\left(\widehat{M}_{\Phi}\right) \triangleleft$. To this end, let $\mathbb{Q} \in \widehat{M}_{\Phi}=M_{\widehat{\Phi}}$ and $X \in C_{\Phi}$. Then $X \in C_{\widehat{\Phi}} \subseteq \overline{K-L_{+}^{1}(\mathbb{Q})} L^{1}(\mathbb{Q})$. Thus there exists a sequence $\left(X_{n}\right)_{n=1}^{\infty} \subseteq K-L_{+}^{1}(\mathbb{Q})$ such that $\left\|X-X_{n}\right\|_{L^{1}(\mathbb{Q})} \rightarrow 0$. Since $\mathbb{Q} \in M_{1}$, it follows that $\mathbb{E}_{\mathbb{Q}}\left[X_{n}\right] \leq 0$ for all $n$ and hence $\mathbb{E}_{\mathbb{Q}}[X] \leq 0$, implying that $C_{\Phi} \subseteq R\left(\widehat{M}_{\Phi}\right)^{\triangleleft}$

Theorem 6.4. If $\Phi$ satisfies the growth condition (4.4) and $M_{\Phi} \neq \emptyset$ then

$$
F_{\Phi} \cap \mathcal{W}\left(R\left(M_{1}\right)\right)=\mathcal{W}\left(R\left(\widehat{M}_{\Phi}\right)\right)={\overline{\mathcal{W}}\left(R\left(M_{\Phi}\right)\right)}^{\sigma\left(F_{\Phi}, E_{\Phi}\right)} .
$$

Moreover,

and

$$
C_{\Phi}=c_{E_{\Phi}}(K)={\overline{K_{\Phi}}}^{\sigma\left(E_{\Phi}, F_{\Phi}\right)}
$$

$$
R\left(\widehat{M}_{\Phi}\right)={\overline{R\left(M_{\Phi}\right)}}^{\sigma\left(F_{\Phi}, E_{\Phi}\right)} .
$$

Proof. Firstly, due to Proposition 4.8 we have

$$
R\left(\widehat{M}_{\Phi}\right)=R\left(\left[M_{\Phi}\right] \cap M_{1}\right)=F_{\Phi} \cap R\left(M_{1}\right),
$$

so that

$$
\mathcal{W}\left(R\left(\widehat{M}_{\Phi}\right)\right)=F_{\Phi} \cap \mathcal{W}\left(R\left(M_{1}\right)\right)
$$

(by Lemma 2.1). Consequently, Lemma 5.2 implies

$$
\mathcal{W}\left(R\left(\widehat{M}_{\Phi}\right)\right)^{\triangleleft}=\left(K_{\Phi}^{\triangleleft}\right)^{\triangleleft}=K_{\Phi}^{\triangleleft \triangleleft},
$$

where the polarisation $\triangleleft$ now is based on the bilinear system $\left(E_{\Phi}, F_{\Phi}\right)$ ! Now, we may apply Proposition 6.3, Proposition 2.6 and Proposition 2.7, and it follows

$$
C_{\Phi}=R\left(\widehat{M}_{\Phi}\right)^{\triangleleft}=\mathcal{W}\left(R\left(\widehat{M}_{\Phi}\right)\right)^{\triangleleft}=K_{\Phi}^{\triangleleft \triangleleft}={\overline{K_{\Phi}}}^{\sigma\left(E_{\Phi}, F_{\Phi}\right)} .
$$

Consequently, Proposition 6.3 leads to

$$
\mathcal{W}\left(R\left(\widehat{M}_{\Phi}\right)\right)=F_{\Phi} \cap \mathcal{W}\left(R\left(M_{1}\right)\right)=K_{\Phi}^{\triangleleft}=C_{\Phi}^{\triangleleft}=R\left(M_{\Phi}\right)^{\triangleleft \triangleleft}={\overline{\mathcal{W}}\left(R\left(M_{\Phi}\right)\right)}^{\sigma\left(F_{\Phi}, E_{\Phi}\right)} .
$$

Since $\mathbb{1}_{\Omega} \in E_{\Phi}$, the functional $F_{\Phi} \ni w \mapsto \mathbb{E}_{\mathbb{P}}[w]$ is $\sigma\left(F_{\Phi}, E_{\Phi}\right)$-continuous, implying that $\left\{w \in F_{\Phi}: \mathbb{E}_{\mathbb{P}}[w]=1\right\}$ is $\sigma\left(F_{\Phi}, E_{\Phi}\right)$-closed. Since

$$
R\left(M_{\Phi}\right)=\mathcal{W}\left(R\left(M_{\Phi}\right)\right) \cap\left\{w \in F_{\Phi}: \mathbb{E}_{\mathbb{P}}[w]=1\right\},
$$


we therefore obtain

$$
\begin{aligned}
{\overline{R\left(M_{\Phi}\right)}}^{\sigma\left(F_{\Phi}, E_{\Phi}\right)} & \subseteq{\overline{\mathcal{W}\left(R\left(M_{\Phi}\right)\right)}}^{\sigma\left(F_{\Phi}, E_{\Phi}\right)} \cap\left\{w \in F_{\Phi}: \mathbb{E}_{\mathbb{P}}[w]=1\right\} \\
& =F_{\Phi} \cap \mathcal{W}\left(R\left(M_{1}\right)\right) \cap\left\{w \in F_{\Phi}: \mathbb{E}_{\mathbb{P}}[w]=1\right\} \\
& =F_{\Phi} \cap R\left(M_{1}\right)=R\left(\widehat{M}_{\Phi}\right) .
\end{aligned}
$$

Remember that the last equality follows from Proposition 4.8. To prove the other inclusion, let $\mathbb{Q}_{1} \in \widehat{M}_{\Phi}$ arbitrary and fix $\mathbb{Q}_{0} \in M_{\Phi}$. Then, due to Lemma 4.6, $\mathbb{Q}^{(n)}:=(1-1 / n) \mathbb{Q}_{1}+1 / n \mathbb{Q}_{0} \in M_{\Phi}$ for any $n \in \mathbb{N}$, and obviously $R\left(\mathbb{Q}^{(n)}\right) \longrightarrow$ $R\left(\mathbb{Q}_{1}\right)$ in the topology $\sigma\left(F_{\Phi}, E_{\Phi}\right)$.

Corollary 6.5. Let $X \in E_{\Phi}$ and assume that $\Phi$ satisfies the growth condition (4.4) and $M_{\Phi} \neq \emptyset$. Then

$$
\bar{\pi}\left(X ; C_{\Phi}\right)=\sup _{\mathbb{Q} \in M_{\Phi}} \mathbb{E}_{\mathbb{Q}}[X]
$$

Proof. Due to Theorem 6.4 we may apply Corollary 5.5 to $M=M_{\Phi}$.

Remark 6.6. If $\Phi$ corresponds to a utility function which is supported on a halfline (i.e., $U:(a, \infty) \rightarrow \mathbb{R}$, where $a>-\infty)$ then the convex conjugate $\Phi$ of $U$ is asymptotically linear as $y \rightarrow \infty$. As mentioned in Remark 4.5 (ii), this means that $\widehat{M}_{\Phi}=M_{1}$. For such utility functions therefore, the set of super-replicable contingent claims does not depend specifically on the shape of $U$. In fact, $C_{\Phi}=C_{i d}$ where $i d(y):=y$, and we recover the polar relations

$$
R\left(M_{1}\right)^{\triangleleft}=C_{i d} \quad \text { and } \quad\left(C_{i d}\right)^{\triangleleft}=\mathcal{W}\left(R\left(M_{1}\right)\right) .
$$

This polarity is of a similar nature to [21, Theorem 3.1], in the sense that it is utility independent.

Remark 6.7. Note that in equation (6.4), we may in fact take the closure in any admissible topology (i. e., in any topology which is stronger than the weak topology $\sigma\left(E_{\Phi}, F_{\Phi}\right)$ and weaker than the Mackey topology $\left.\tau\left(E_{\Phi}, F_{\Phi}\right)\right)$. See $[17, \S 98$ and $\S 103]$ for an explanation of the details.

\section{The special case of admissible trading strategies}

In this section we consider the particular case where $K=K^{\text {adm }}$ is the wedge of attainable terminal wealths resulting from zero-financed admissible trading strategies. As an application of our general framework, we show that in this case every contingent claim in $C_{\Phi}=: C_{\Phi}^{\text {adm }}$ even can be approximated by bounded contingent claims which are dominated by terminal wealths in $K^{\mathrm{adm}}$. This approximation is given with respect to the $\left(K^{\mathrm{adm}}-\right.$ related $)$ weak topology $\sigma\left(E_{\Phi}, F_{\Phi}\right)$.

By

$$
\begin{aligned}
C^{\mathrm{adm}} & :=\left(K^{\mathrm{adm}}-L_{+}^{0}\right) \cap L^{\infty}(\mathbb{P}) \\
& =\left\{X \in L^{\infty}(\mathbb{P}): X \leq \widetilde{X} \text { for some } \widetilde{X} \in K^{\mathrm{adm}}\right\}
\end{aligned}
$$


we denote the wedge of all a.s bounded contingent claims that can be dominated by a terminal wealth in $K^{\mathrm{adm}}$. Since $K^{\mathrm{adm}} \subseteq K_{\Phi}^{\mathrm{adm}}$, it follows that

$$
C^{\mathrm{adm}} \subseteq\left(K^{\mathrm{adm}}-L_{+}^{0}\right) \cap L^{1}(\mathbb{Q}) \subseteq K^{\mathrm{adm}}-L_{+}^{1}(\mathbb{Q})
$$

for any $\mathbb{Q} \in M_{\Phi}$ and thus

$$
C^{\mathrm{adm}} \subseteq K_{\Phi}^{\mathrm{adm}} \subseteq C_{\Phi}^{\mathrm{adm}} \subseteq E_{\Phi}
$$

A benefit of using admissible strategies is the following approximation result (which follows from Lebesgue's Monotone Convergence Theorem):

Lemma 7.1. Let $X \in K^{\mathrm{adm}}$. Then there exists a constant $c \geq 0$ and a sequence $\left(X_{n}\right)_{n \in \mathbb{N}} \subseteq C^{\text {adm }}$ such that for any probability measure $\mathbb{Q} \ll \mathbb{P}$ the following properties hold:

(i) $-c \leq X_{1} \leq X_{2} \leq \ldots \leq X_{n} \uparrow X \quad \mathbb{Q}$-a.s;

(ii) $-c \leq \lim _{n \rightarrow \infty} \mathbb{E}_{\mathbb{Q}}\left[X_{n}\right]=\mathbb{E}_{\mathbb{Q}}[X] \in \mathbb{R} \cup\{\infty\}$.

As a consequence of Lemma 7.1 we may substitute the wedge $K^{\text {adm }}$ by the wedge $C^{\text {adm }} \subseteq L^{\infty}(\mathbb{P})$ in equation (4.2) to get

$$
R\left(M_{1}^{\mathrm{adm}}\right)=\left\{Y \in L_{+}^{1}(\mathbb{P}): \mathbb{E}_{\mathbb{P}}[Y]=1 \text { and } \mathbb{E}_{\mathbb{P}}[X Y] \leq 0 \text { for all } X \in C^{\text {adm }}\right\} .
$$

Theorem 7.2. If $\Phi$ satisfies the growth condition (4.4) and $M_{\Phi}^{\mathrm{adm}} \neq \emptyset$ then

$$
C_{\Phi}^{\mathrm{adm}}={\overline{C^{\mathrm{adm}}}}^{\sigma\left(E_{\Phi}, F_{\Phi}\right)}
$$

Proof. Due to equation (7.1) and Proposition 4.8, $\left(C^{\text {adm }}\right)^{\triangleleft} \subseteq F_{\Phi} \cap \mathcal{W}\left(R\left(M_{1}^{\text {adm }}\right)\right)=$ $\mathcal{W}\left(R\left(\widehat{M_{\Phi}^{\text {adm }}}\right)\right)$. Hence, as a result of Proposition 6.3 and Proposition 2.7,

$$
{\overline{C^{\mathrm{adm}}}}^{\sigma\left(E_{\Phi}, F_{\Phi}\right)} \subseteq C_{\Phi}^{\mathrm{adm}}=\left(\mathcal{W}\left(R\left({\widehat{M_{\Phi}^{\mathrm{adm}}}}^{\mathrm{ad}}\right)\right)^{\triangleleft} \subseteq\left(C^{\mathrm{adm}}\right)^{\triangleleft \triangleleft}={\overline{C^{\mathrm{adm}}}}^{\sigma\left(E_{\Phi}, F_{\Phi}\right.}\right) .
$$

\section{Appendix}

Let us recall an important version of the Hyperplane Separation Theorem in finite-dimensional vector spaces which is not only known as one of the main building blocks for duality theorems in linear programming. It also has other numerous applications, e.g., to the Karush-Kuhn-Tucker theorem in nonlinear programming and zero-sum games in economic theory (cf. [8]).

Theorem 8.1 (Farkas' Lemma). Let $m, n \in \mathbb{N}, A \in M\left(m \times n ; \mathbb{R}^{n}\right)$ and $b \in \mathbb{R}^{m}$. Then either

(i) there exists $x \in \mathbb{R}^{n}$ such that $x \geq 0$ and $A x=b$

or

(ii) there exists $y \in \mathbb{R}^{m}$ such that $\langle y, b\rangle>0$ and $A^{\top} y \leq 0$. 
Theorem 8.1 implies the non-trivial fact that the finitely generated wedge $A\left(\mathbb{R}_{+}^{n}\right)$ is closed in $\mathbb{R}^{m}$. More generally, the following statement, which transfers Theorem 8.1 to infinite-dimensional Banach lattices, shows that Farkas' Lemma even is equivalent to the closedness of the wedge $A\left(\mathbb{R}_{+}^{n}\right)$ ! Concerning the basics of Banach lattices and positive operators, we refer the reader to [32].

Theorem 8.2. Let $E, F$ be arbitrary Banach lattices and $A: E \longrightarrow F$ be a continuous linear operator. Then the following statements are equivalent:

(i) $A\left(E_{+}\right)$is $\sigma\left(F, F^{\prime}\right)$-closed;

(ii) Let $b \in F$. Then either

(1) there exists $x \in E_{+}$such that $A x=b$

or

(2) there exists $y^{\prime} \in F^{\prime}$ such that $\left\langle b, y^{\prime}\right\rangle>0$ and $A^{\prime} y^{\prime} \leq 0$.

Proof. First assume that $(i)$ holds. We want to show $(i i)$. To this end, we consider the wedge

$$
G:=\left(\left(A^{\prime}\right)^{-1}\left(-E_{+}^{\prime}\right)\right)^{\triangleleft}=\left\{b \in F:\left\langle b, y^{\prime}\right\rangle \leq 0 \text { for all } y^{\prime} \in\left(A^{\prime}\right)^{-1}\left(-E_{+}^{\prime}\right)\right\} \subseteq F .
$$

Since $F \backslash G$ exactly consists of all $b \in F$, satisfying condition (2) in (ii) above, we only have to show that

$$
A\left(E_{+}\right)=G .
$$

Obviously, by definition of $G, A\left(E_{+}\right) \subseteq G$. Let $y^{\prime} \in\left(A\left(E_{+}\right)\right)^{\triangleleft}$. Then $\left\langle x, A^{\prime} y^{\prime}\right\rangle=\left\langle A x, y^{\prime}\right\rangle \leq 0$ for any $x \in E_{+}$. Hence, $y^{\prime} \in\left(A^{\prime}\right)^{-1}\left(-E_{+}^{\prime}\right)$. Thus, $\left(A\left(E_{+}\right)\right)^{\triangleleft} \subseteq\left(A^{\prime}\right)^{-1}\left(-E_{+}^{\prime}\right)$ and consequently,

$$
A\left(E_{+}\right) \subseteq G \subseteq\left(A\left(E_{+}\right)\right) \triangleleft \triangleleft .
$$

Due to Proposition 2.7 and the assumed weak closedness of the wedge $A\left(E_{+}\right)$, it follows that

$$
\left(A\left(E_{+}\right)\right)^{\triangleleft \triangleleft}={\overline{\mathcal{W}\left(A\left(E_{+}\right)\right)}}^{\sigma\left(F, F^{\prime}\right)}={\overline{A\left(E_{+}\right)}}^{\sigma\left(F, F^{\prime}\right)}=A\left(E_{+}\right),
$$

Hence, we obtain equality (8.1).

Assume now that statement $(i i)$ is true. We want to show $(i)$. To this end, let $b \in{\overline{A\left(E_{+}\right)}}^{\sigma\left(F, F^{\prime}\right)}$. Then there exists a net $\left(x_{\alpha}\right) \subseteq E_{+}$such that $b$ is the $\sigma\left(F, F^{\prime}\right)$-limit of the net $\left(A x_{\alpha}\right)$. Assume by contradiction that $b \notin A\left(E_{+}\right)$. Then, due to assumption ( $i i)$, condition (2) must be true (since (1) is false). Thus, there exists a continuous linear functional $y^{\prime} \in F^{\prime}$ such that for any $x \in E_{+}$we have

$$
\left\langle A x, y^{\prime}\right\rangle=\left\langle x, A^{\prime} y^{\prime}\right\rangle \leq 0<\left\langle b, y^{\prime}\right\rangle \text {. }
$$

In particular, $\left\langle b, y^{\prime}\right\rangle=\lim _{\alpha}\left\langle A x_{\alpha}, y^{\prime}\right\rangle \leq 0$ - a contradiction. Consequently, $b \in A\left(E_{+}\right)$.

\section{Acknowledgements}

The authors would like to thank an anonymous referee for some useful and helpful comments on this paper. 


\section{References}

[1] C.D. Aliprantis, O. Burkinshaw, Positive Operators. Springer, Netherlands (2006).

[2] C.D. Aliprantis, R. Tourky, Cones and Duality. AMS, Graduate Studies in Mathematics, vol. 84 (2007).

[3] P. Artzner, F. Delbaen, J.-M. Eber, D. Heath, Coherent measures of risk. Math. Finance, 9(3), 203-228 (1999).

[4] M. Baxter, A. Rennie. Financial Calculus: An Introduction to Derivative Pricing. Cambridge University Press, Cambridge (1996).

[5] S. Biagini, M. Frittelli, On the super-replication price of unbounded claims. Ann. Appl. Prob., 14(4), 1970-1991 (2004).

[6] S. Biagini, M. Frittelli, Utility maximization in incomplete markets for unbounded processes. Finance Stochast., 9, 493-517 (2005).

[7] S. Biagini, M. Frittelli, A unified framework for utility maximization problems: an Orlicz space approach. Ann. Appl. Prob., 18(3), 929-966 (2008).

[8] J. Borwein, A.S. Lewis, Convex Analysis and Nonlinear Optimization. Springer, CMS Books in Mathematics, 2nd edn (2006).

[9] F. Delbaen, W. Schachermayer, A general version of the fundamental theorem of asset pricing. Math. Ann., 300, 463-520 (1994).

[10] F. Delbaen, W. Schachermayer, The no-arbitrage property under a change of numéraire. Stoch. Stoch. Rep., 53, 213-226 (1995).

[11] F. Delbaen, W. Schachermayer, The fundamental theorem of asset pricing for unbounded stochastic processes. Math. Ann., 312, 215-250 (1998).

[12] F. Delbaen, W. Schachermayer, Applications to mathematical finance. In: Handbook of the geometry of Banach spaces, vol. 1, North-Holland, Amsterdam, pp. 369-391 (2001).

[13] F. Delbaen, W. Schachermayer, The Mathematics of Arbitrage. Springer Finance (2006).

[14] R.J. Elliott, P.E. Kopp, Mathematics of Financial Markets. Springer Finance, 2nd edn (2005).

[15] M. Frittelli, Optimal solutions to utility maximization and to the dual problem. Preprint (2000).

[16] M. Frittelli, E. Rosazza Gianin, Equivalent formulations of reasonable asymptotic elasticity. Preprint (2002).

[17] H.G. Heuser, Functional Analysis. John Wiley \& Sons, New York (1982).

[18] J. Hugonnier, D. Kramkov, Optimal investment with random endowments in incomplete markets. Ann. Appl. Prob., 14, 845-864 (2004).

[19] H. Jarchow, Locally convex spaces. B. G. Teubner, Stuttgart (1981).

[20] F.C. Klebaner, Introduction to Stochastic Calculus with Applications. Imperial College Press (1998).

[21] D. Kramkov, W. Schachermayer, The asymptotic elasticity of utility functions and optimal investment in incomplete markets. Ann. Appl. Prob., 9, 904-950 (1999).

[22] W.A.J. Luxemburg, A.C. Zaanen, Riesz Spaces I. North-Holland, Amsterdam (1971).

[23] P. Meyer-Nieberg, Banach Lattices. Springer, Berlin (1991). 
[24] M.P. Owen, On utility based super-replication prices. Preprint (2003).

[25] M.P. Owen, F. Oertel, On utility-based super-replication prices of contingent claims with unbounded payoffs. J. Appl. Prob., 44(4), 880-888 (2007).

[26] M.P. Owen, G. Žitković, Optimal investment with an unbounded random endowment and utility-based pricing methods. To appear in Math. Finance (2008).

[27] P. Protter. Stochastic Integration and Differential Equations, 2nd edn. Springer, Berlin (2003).

[28] R.T. Rockafellar, Convex Analysis. Princeton University Press (1972).

[29] S. Roman, Introduction to the Mathematics of Finance. Springer Undergraduate Texts in Mathematics (2004).

[30] W. Schachermayer, Optimal investment in incomplete markets when wealth may become negative. Ann. Appl. Prob., 11, 694-734 (2001).

[31] W. Schachermayer, Utility maximisation in incomplete markets. Stochastic Methods in Finance. Lectures given at the CIME-EMS Summer School in Bressanone/Brixen, Italy, July 6-12, 2003, M. Frittelli, W. Runggaldier, (eds). Springer Lecture Notes in Mathematics 1856, 225-288 (2004).

[32] H.H. Schaefer, Banach Lattices and Positive Operators. Springer (1974).

[33] Y.-C. Wong, Some Topics in Functional Analyis and Operator Theory. Science Press (1993).

Frank Oertel

Department of Mathematics

University College Cork

Mark P. Owen

Department of Actuarial Mathematics and Statistics

Maxwell Institute for Mathematical Sciences and Heriot-Watt University

Received 14 November 2007; accepted 26 August 2008

To access this journal online: www.birkhauser.ch/pos 